\title{
AVERAGE DISTANCES IN COMPACT CONNECTED SPACES
}

\author{
David Yost
}

We give a simple proof of the fact that compact, connected topological spaces have the "average distance property". For a metric space $(X, d)$, this asserts the existence of a unique number $a=a(X)$ such that, given finitely many points $x_{1}, \ldots, x_{n} \in X$, then there is some $y \in X$ with

$$
\frac{1}{n} \sum_{i=1}^{n} d\left(y, x_{i}\right)=a \text {. }
$$

We examine the possible values of $a(X)$, for subsets of finite dimensional normed spaces. For example, if diam $(X)$ denotes the diameter of some compact, convex set in a euclidean space, then $a(X) \leq \operatorname{diam}(X) / \sqrt{2}$. On the other hand, $a(X) / \operatorname{diam}(X)$ can be arbitrarily close to 1 , for non-convex sets in euclidean spaces of sufficiently large dimension.

\section{The Gross-Stadje theorem}

In [7], Stadje proved the following interesting result.

THEOREM 1. Let $X$ be a compact, connected topological space and $d: x^{2} \rightarrow \mathbf{R}$ a continuous, symnetric function. Then there is a unique number $a=a(X, d)$ with the following property: for alz $n \in N$, and for

Received 5 May 1982. The author is very grateful to Graham Elton, Sid Morris and John Strantzen for a number of stimulating discussions, and to Ian Robinson for some calculations. 
all $x_{1}, \ldots, x_{n} \in X$, there is a point $y \in X$ such that

$$
\frac{1}{n} \sum_{i=1}^{n} d\left(x_{i}, y\right)=a
$$

Typically, $d$ will be a metric on $X$, although that assumption is not necessary. Bearing this in mind, the property characterizing $a(X, d)$ is called the "average distance property". For the special case when $d$ is a metric, Theorem 1 had previously been proved by Gross [1]. In the general case, we will call $a(X, d)$ the Gross-Stadje number for $(X, d)$.

One purpose of this note is to present a simple proof of the GrossStadje Theorem. Our proof of the existence of the Gross-Stadje number is new and completely elementary. First, it will be helpful to introduce some notation.

Thet $F=\bigcup_{n=1}^{\infty} X^{n}$. Thus $F$ is the set of all ordered finite-tuples, with pembers from $X$. If $x \in X$, and $F=\left(x_{1}, \ldots, x_{n}\right) \in F$, put $d(x, F)=\frac{1}{n} \sum_{i=1}^{n} d\left(x, x_{i}\right) \cdot$ Then put $\alpha_{F}=\inf \{d(x ; F): x \in X\} \quad$ and $\beta_{F}=\sup \{d(x, F): x \in X\}$.

We claim that $\alpha_{F} \leq B_{G}$ whenever $F, G \in F$. Let us write $F=\left(x_{1}, \ldots, x_{m}\right)$ and $G=\left(y_{1}, \ldots, y_{n}\right)$. It suffices to show that, for some $i \leq n$ and some $j \leq m$, we have $d\left(y_{i}, F\right) \leq d\left(x_{j}, G\right)$. Suppose that this is not true. Then $d\left(y_{i}, F\right)>d\left(x_{j}, G\right)$, for all $i \leq n, j \leq m$. summing over $i$ and $j$ then yields

$$
m \sum_{i=1}^{n} d\left(y_{i}, F\right)>n \sum_{j=1}^{m} d\left(x_{j}, G\right) \text {. }
$$

Since $d$ is symmetric, both sides of this inequality are equal to $\sum_{i=1}^{n} \sum_{j=1}^{m} d\left(y_{i}, x_{j}\right)$. This is a contradiction, so our claim must be correct.

Now existence of $a(X, d)$ can be easily proved. What we wish to show is that $(\exists ! a \in \mathbf{R})(\forall F \in F)(a \in\{d(x, F): x \in X\})$. For any $F \in F$, the 
map $x \mapsto d(x, F)$ is a continuous function on $X$. Since $X$ is compact and connected, $\{d(x, F): x \in X\}$ must be the closed interval $\left[\alpha_{F}, \beta_{F}\right]$. The conclusion of Theorem $I$ then becomes $(\exists ! a)(\forall F \in F)\left(\alpha_{F} \leq a \leq \beta_{F}\right)$. Our previous claim tells us that $\sup \left\{\alpha_{F}: F \in F\right\} \leq \inf \left\{\beta_{F}: F \in F\right\}$.

Existence of $a$ follows immediately.

It is a little harder to prove the uniqueness of $a$. First note that each $F \in F$ induces, iin a natural way, an atomic probability measure on $X$. We will use the same symbol for the probability measure and the ordered tuple; thus $d(x, F)=\int_{X} d(x, y) d F(y)$. Let $P$ denote the set of all regular Borel probability measures on $X$, equipped with the vague topology. (For details of the relationship between $P$ and $X$, we refer the reader to [4, section 22A].) In this topology, a net $\dot{P}_{\alpha}$ is convergent to $P$ if and only if $\int_{X} f(x) d P_{\alpha}(x) \rightarrow \int_{X} f(x) d P(x)$, for every continuous function $f: X \rightarrow \mathbf{R}$. Then $P$ is a compact, convex set; and it can be deduced from the Krein-Milman theorem [4, Section 13B] that $F$ is dense in $P$. If $F_{\alpha} \rightarrow P$ vaguely, and $x_{\alpha} \rightarrow x$ in $X$, it is a routine exercise to show that $d\left(x_{\alpha}, F_{\alpha}\right) \rightarrow d(x, P)$. From these facts it follows that

$$
\underline{v}=\sup _{F \in F} \min _{x \in X} d(x, F)=\max _{P \in P} \min _{x \in X} d(x, P)
$$

and

$$
\bar{v}=\inf _{F \in F} \max _{x \in X} d(x, F)=\min _{P \in P} \max _{x \in X} d(x, P)
$$

We have already shown that $\underline{v} \leq \bar{v}$. A generalization of Ville's version of the minimax theorem [6, p. 69] tells us that $\underline{v}=\bar{v}$. Thus $\sup _{F \in F} \alpha_{F}=\inf _{F \in F} \beta_{F}$, and so $a$ is unique. This completes our proof of Theorem 1 .

Graham Elton has pointed out (private communcation) that $(X, d)$ will have the following strong version of the average distance property: given any regular, Borel probability measure $P$ on $X$, there is a point $x \in X$ with $d(x, P)=a(X, d)$. This result follows from the last paragraph. 
It also follows from the proof above that there are two probability measures, $P$ and $Q$, on $X$ such that $d(x, P) \leq a(X, d) \leq d(x, Q)$ for all $x \in X$. Joan Cleary and Sid Morris (private communication) have used this idea to calculate the Gross-Stadje numbers of regular polygons.

If there is a single probability measure $P$, on $X$, such that $d(x, P)$ is independent of $x$, then $a(X, d)$ is easy to determine. We only have to calculate $d(x, P)$ for a convenient point $x \in X$. Morris and Nickolas [5] have used this to evaluate the Gross-Stadje numbers of sufficiently symmetric metric spaces, such as spheres.

Sometimes it is difficult to find $a(X, d)$ exactly, and we must settle for some sort of estimate. In such a situation, the following result might be useful.

PROPOSITION 2. Fix $\alpha, \beta \in \mathbb{R}$ with $\alpha \leq \beta$.

(i) Suppose $(X, d)$ has the following property: given any $F \in F$, there is a point $y \in X$ with $\alpha \leq d(y, F) \leq \beta$. Then $\alpha \leq a(X, d) \leq \beta$.

(ii) Suppose there is a point $y \in X$ such that $\alpha \leq d(x, y) \leq \beta$ for all $x \in X$. Then $\alpha \leq \alpha(X, d) \leq \beta$.

Proof. (i) The hypothesis clearly implies that

$$
\sup _{F} \min _{x} d(x, F) \leq \beta \text { and } \alpha \leq \inf _{F} \max d(x, F) .
$$

(ii) This follows immediately from $(i) . \quad / /$

\section{The range of values for metric spaces}

From now on, $(X, d)$ will be a compact, connected metric space, and $a(X, d)$ will be abbreviated to $a(X)$. We assume further that $X$ is not a singleton, and so has strictly positive diameter, $\operatorname{diam}(X)$. Normalizing, we define the dispersion number of $X$ by $m(X)=a(X) / \operatorname{diam}(X)$. It can then be shown $[7, \mathrm{p} .277]$ that $\frac{1}{2} \leq m(X)<1$.

In this section, we will consider the range of values that $m(X)$ may take. The dispersion number gives us some information about how 'spread out' a space is, although perhaps not as much information as we would like. If $m(X)$ is close to 1 , then the points of $X$ are, generally speaking, far apart from one another. The converse is not true. It is possible that $m(X)=\frac{1}{2}$, for a space $X$ which we might intuitively describe as fairly 
spread out.

PROPOSITION 3. Suppose that $m(X)>\frac{1}{2}$. Then there is a metric space $Y$, obtained from $X$ by gluing on a line segment, for which $m(Y)=\frac{1}{2}$.

Proof. Let $I^{\infty}$ denote the space of all sequences from $[0,1]$, equipped with the metric $d\left(\left(\alpha_{n}\right),\left(\beta_{n}\right)\right)=\sup _{n=1}^{\infty}\left|\alpha_{n}-\beta_{n}\right|$. Assume without loss of generality that $\operatorname{diam}(X)=1$. Since $X$ is separable, we may suppose that it is embedded in $I^{\infty}$. Let $a \in I^{\infty}$ be the constant sequence $\left(\frac{1}{2}, \frac{1}{2}, \ldots\right)$. Since $X$ is compact, there is a point $x \in X$ such that $d(a, x)$ is a minimum; that is, $d(a, x)=d(a, X)$. Let $Y=X v[x, a]$, where $[x, a]$ is the line segment joining $x$ to $a$. Then $Y$ is certainly a compact, connected set. Since $x$ is a closest point (in $X$ ) to $a, Y \backslash X$ is just the half-open segment $(x, a]$. Moreover $d(a, y) \leq \frac{1}{2}$ for all $y \in I^{\infty}$, and hence for all $y \in Y$. It follows from Proposition 2 that $a(Y) \leq \frac{1}{2}$. Clearly $\operatorname{diam}(Y)=1$, and thus $m(Y)=\frac{1}{2} \cdot \quad / /$

If $(S, d)$ is any metric space, let $H(S)$ denote the family of all compact, connected, non-empty subsets of $S$. We can turn $H(S)$ into a metric space as follows. For any $X, Y \in H(S)$, define $\rho(X, Y)=\sup \{d(x, Y): x \in X\}$, and $d_{H}(X, Y)=\max \{\rho(X, Y), \rho(Y, X)\}$. Then $d_{H}$ is a metric for $H(S)$ [2, Section 28]. If $S$ is compact, then so is $H(S)$ under this metric.

PROPOSITION 4. For any metric space $(S, d)$, the map $a: H(S) \rightarrow \mathbb{R}$ is continuous. More precisely, we have

$$
|a(X)-a(Y)| \leq \rho(X, Y)+\rho(Y, X) \leq 2 d_{H}(X, Y)
$$

for all $X, Y \in H(S)$.

Proof. Fix $X, Y \in H(S)$, and put $\delta_{1}=\rho(X, Y)$ and $\delta_{2}=\rho(Y, X)$. Let $x_{1}, \ldots, x_{n}$ be any points in $X$. Then there are points $y_{1}, \ldots, y_{n} \in Y$ with $d\left(x_{i}, y_{i}\right) \leq \delta_{1}$ for each $i$. Determine $y \in Y$ so so that $a(y)=\frac{1}{n} \sum_{i=1}^{n} d\left(y, y_{i}\right)$. Then there is a point $x \in X$ with 
$d(x, y) \leq \delta_{2}$. Routine calculations then show that

$$
a(Y)-\delta_{1}-\delta_{2} \leq \frac{1}{n} \sum_{i=1}^{n} d\left(x, x_{i}\right) \leq a(y)+\delta_{1}+\delta_{2} .
$$

An application of Proposition 2 yields

$$
a(Y)-\delta_{1}-\delta_{2} \leq a(X) \leq a(Y)+\delta_{1}+\delta_{2} \cdot \quad / /
$$

THEOREM 5. Let $E$ be any finite dimensional normed space. Then there is a constant $k=k(E)<1$ such that $m(X) \leq k$ whenever $X$ is a compact connected subset of $E$. Moreover,

$$
\{m(X): X \text { is a compact, connected subset of } E\}
$$

is the whole interval $\left[\frac{1}{2}, k(E)\right]$.

Proof: It suffices to show that $a(X) \leq k$ whenever $\operatorname{diam}(X)=1$. Clearly any set of diameter 1 is isometric to a subset of $S$, the closed unit ball of $E$. So let $T=\{X \in H(S): \operatorname{diam}(X)=1\}$. Then $T$ is a compact, metric space, $a: T \rightarrow \mathrm{R}$ is continuous, and $a(X)<1$ for every $X \in T$. It follows that $k(E)=\max \{a(X): X \in T\}<1$.

Finally, choose $X \in T$ so that $a(X)=m(X)=k(E)$, and let $Y$ be a line segment with length 1 . If $0 \leq \lambda \leq 1$, then

$$
\lambda X+(1-\lambda) Y=\{\lambda x+(1-\lambda) y: x \in X, y \in Y\}
$$

is a compact, connected subset of $E$, and is clearly not a singleton. Thus we can define a continuous map $[0,1]+\mathbb{R}$ by $\lambda \mapsto m(\lambda X+(1-\lambda) Y)$. Since $0 \mapsto \frac{1}{2}$ and $I \mapsto k(E)$, the intermediate value theorem finishes the proof. //

Let us define $k_{n}=\sup \{k(E): E$ is an $n$-dimensional normed space $\}$. It is almost obvious that $k_{1}=\frac{1}{2}$. It would be interesting to know* whether $k_{n}<1$ for $n=2,3, \ldots$. The next result shows that $k_{n} \rightarrow 1$ as $n \rightarrow \infty$.

THEOREM 6. If $X$ is a compact, convex set in some n-dimensional normed space, then $m(X) \leq n /(n+1)$. This estimate is sharp.

Proof. For each $x \in X$, let $A(x)=(1 /(n+1))(x+n X)$. Then each

* See note added in proof. 
$A(x)$ is a compact, convex subset of $X$. If $x_{1}, x_{2}, \ldots, x_{n+1}$ are any elements of $X$, it is easy to verify that $(1 /(n+1)) \sum_{i=1}^{n+1} x_{i} \in \prod_{i=1}^{n+1} A\left(x_{i}\right)$. We can then deduce from Helly's theorem [3], the existence of some $a \in \cap A(x)$. Now fix $x \in X$. Since $a \in A(x)$, there is a $y \in X$ with $x \in X$

$a=(1 /(n+1))(x+n y)$. Then $\|x-a\|=(n /(n+1))\|x-y\| \leq(n /(n+1)) \operatorname{diam}(X)$ and so $a(X) \leq(n /(n+1)) \operatorname{diam}(X)$.

To see that this estimate is sharp, give $\mathbb{R}^{n+1}$ the $z_{1}$-norm, $\left\|\left(\alpha_{0}, \alpha_{1}, \ldots, \alpha_{n}\right)\right\|=\sum_{i=0}^{n}\left|\alpha_{i}\right|$, and let $x$ be the convex hull of $F=\left\{e_{0}, e_{1}, e_{2}, \ldots, e_{n}\right\}$. Then $X$ is contained in the $n$-dimensional affine subspace $\left\{\left(\alpha_{0}, \alpha_{1}, \ldots, \alpha_{n}\right): \sum_{i=0}^{n} \alpha_{i}=1\right\}$. Routine calculations show that $d(x, F)=2 n /(n+1)$, for any $x \in X$. Since $\operatorname{diam}(X)=2$, it follows that $m(X)=n /(n+1)$. //

\section{Subsets of euclidean spaces}

Stadje claims [7, p. 278] that if $X$ is a compact, convex subset of the euclidean space $\mathbf{R}^{n}$, then $m(X) \leq \frac{1}{2} \sqrt{5-2 \sqrt{3}}$. Recently, Strantzen [8] has shown that $m(X) \leq \sqrt{n /(2 n+2)}$ for any such $X$, and that this bound is sharp. This improves Stadje's estimate for $n=2$ and $n=3$, and disproves his claim for $n \geq 4$. However, we still have the uniform estimate $m(X) \leq 1 / \sqrt{2}$, whenever $X$ is a compact, convex subset of some euclidean space. Theorem 6 shows that no such uniform bound exists for non-euclidean spaces.

It is still of interest to know whether there is such a uniform bound, for non-convex sets in euclidean spaces. There is not; we will see from Theorem 9 that $k\left(\mathbf{R}^{n}\right) \rightarrow 1$ as $n \rightarrow \infty$. The next two results help to identify those sets with large dispersion numbers.

THEOREM 7. Let $X$ be a compact, connected subset of some normed space. Let $Y$ be a closed, connected subset of $X$, and suppose that the convex hull of $Y$ contains $X$. Then $m(X) \leq m(Y)$. 
Proof. Clearly $\operatorname{diam}(Y)=\operatorname{diam}(X)$, so we need show only that $a(X) \leq a(Y)$. Let $F$ be any finite ordered-tuple from $Y$. Then $F$ is also a finite ordered-tuple from $X$, so $d(x, F)=a(X)$ for some $x \in X$. However, $x=\sum_{i} \lambda_{i} y_{i}$, for some $y_{i} \in Y, \lambda_{i} \geq 0$ with $\sum_{i} \lambda_{i}=1$. Then $a(X)=d\left(\sum_{i} \lambda_{i} y_{i}, F\right) \leq \sum_{i} \lambda_{i} d\left(y_{i}, F\right)$, and so $a(X) \leq d\left(y_{i}, F\right)$, for at least one value of $i$. It follows from Proposition 2 that $a(X) \leq a(Y)$.

COROLLARY 8. Let $X$ be a compact, connected subset of a finite dimensional normed space, whose boundary $\partial X$ is connected. Then $m(X) \leq m(\partial X)$.

Proof. It follows from the separation theorem that $X$ is contained in the convex hull of $\partial X$. //

Corollary 8 was first proved by Graham Elton for finite dimensional euclidean spaces.

Let $S^{n}$ denote, as usual, the surface of the unit ball in $\mathbb{R}^{n+1}$. Graham Elton, Sid Morris and Peter Nickolas (private communication) have shown that the sequence $m\left(s^{n}\right)$ increases monotonically, and has limit $1 / \sqrt{2}$. Given Corollary 8 , it is then tempting to conjecture that $m(X) \leq 1 / \sqrt{2}$, whenever $X$ is contained in a finite dimensional euclidean space. The truth is quite different.

THEOREM 9. There exist compact, connected sets $X_{n} \subset \mathbb{R}^{n}$ such that $m\left(X_{n}\right)+1$ as $n \rightarrow \infty$. More precisely, $X_{n}$ can be chosen so that $m\left(x_{n}\right) \geq n /(n+1)$. (obviously $x_{n}$ cannot be convex.)

Proof. In $\mathbb{R}^{n+1}$, let $F_{n}$ be the finite set $\left\{e_{i} / \sqrt{2}: 0 \leq i \leq n\right\}$. For $0 \leq j<k \leq n$, let $A(j, k)$ be the arc with centre at $(1 /((n-1) \sqrt{2}))\left(\sum_{i=0}^{n} e_{i}-e_{j}-e_{k}\right)$, which joins $e_{j} / \sqrt{2}$ to $e_{k} / \sqrt{2}$. Then $A(j, k)$ has radius $\sqrt{n /(2 n-2)}$, and parameterization 


$$
\begin{aligned}
& x_{j}(\theta)=\frac{\cos \theta}{\sqrt{2}}-\frac{\sin \theta}{\sqrt{2\left(n^{2}-1\right)}}, \\
& x_{k}(\theta)=\frac{n \sin \theta}{\sqrt{2\left(n^{2}-1\right)}}, \\
& x_{i}(\theta)=\frac{1-\cos \theta}{\sqrt{2}(n-1)}-\frac{\sin \theta}{\sqrt{2\left(n^{2}-1\right)}}, \text { for } i \neq j, k,
\end{aligned}
$$

where $0 \leq \theta \leq \operatorname{arcos}(1 / n)$. Then $X_{n}=U\{A(j, k): 0 \leq j \leq k \leq n\}$ is a compact, connected subset of the n-dimensional affine subspace $\left\{\left(x_{0}, \ldots, x_{n}\right): \sum_{i=0}^{n} x_{i}=\sqrt{2}\right\}$. It is routine to show that $\left\|x-e_{i} / \sqrt{2}\right\|=1$, whenever $x \in A(j, k)$ and $j \neq i \neq k$. Thus, for any $x \in X$,

$$
\begin{aligned}
d\left(x, F_{n}\right) & =\frac{1}{n+1} \sum_{i=0}^{n}\left\|x-e_{i} / \sqrt{2}\right\| \\
& =\frac{1}{n+1}\left(n-1+\left\|x-e_{j} / \sqrt{2}\right\|+\left\|x-e_{k} / \sqrt{2}\right\|\right) \text { for suitable } j, k \\
& \geq \frac{1}{n+1}\left(n-1+\left\|e_{j} / \sqrt{2}-e_{k} / \sqrt{2}\right\|\right) \\
& =\frac{n}{n+1} .
\end{aligned}
$$

It follows that $a\left(x_{n}\right) \geq n /(n+1)$. When $n=2, x_{n}$ is the well-known Reuleaux triangle, and it is easy to see that $\operatorname{diam}\left(x_{2}\right)=1$. Thus $m\left(X_{2}\right) \geq 2 / 3$.

Unfortunately, it is not true that $\operatorname{diam}\left(x_{n}\right)=1$ for $n \geq 3$. One can show that $\|x-y\|$ is a maximum (over $x, y \in X_{n}$ ) when $x$ and $y$ are the midpoints of two arcs which do not share a common vertex. It follows that $\operatorname{diam}\left(X_{n}\right)=(\sqrt{n(n+1)}-\sqrt{2}) /(-1) \rightarrow 1$ as $n \rightarrow \infty$. Thus $m\left(X_{n}\right) \rightarrow 1$ as $n \rightarrow \infty$.

If $Y_{n}$ is any closed, connected subset of $X_{n}$ which contains $F_{n}$, the same reasoning shows that $a\left(y_{n}\right) \geq n /(n+1)$ and $\operatorname{diam}\left(Y_{n}\right) \rightarrow 1$. For 
example, we could choose

$$
\begin{aligned}
& \text { (i) } y_{n}=U\{A(i-1, i): 1 \leq i \leq n\} \text {, or } \\
& \text { (ii) } Y_{n}=A(0, n) \cup U\{A(i-1, i): 1 \leq i \leq n\} \text {, or } \\
& \text { (iii) } Y_{n}=U\{A(0, i): 1 \leq i \leq n\} .
\end{aligned}
$$

In case (i), $Y_{n}$ will be homeomorphic to a line segment, and in case (ii), $y_{n}$ will be homeomorphic to a circle. For the last choice, it is possible to show that $\operatorname{diam}\left(Y_{n}\right)=1$ and so $m\left(Y_{n}\right) \geq n /(n+1)$.

Some numerical calculations show that the dispersion number for the Reuleaux triangle is 0.668 (to three significant figures). It follows that $k\left(R^{2}\right) \geq 0.668$. Graham Elton (private communication) has shown that $k\left(\mathbf{R}^{2}\right) \leq 0.775$. It would be interesting to narrow this gap.

ADDED IN PROOF (25 June 1982). We have recently shown that $k_{n}<1$. A sketch of the proof follows.

If $\|\cdot\|$ is any norm on $\mathbb{R}^{n}$, let $v$ denote its restriction to $I^{n}=[-1,1]^{n}$, and also the derived metric. If $E$ is any $n$-dimensional normed space, Auerbach's Lemma [J. Lindenstrauss and L. Tzafriri, Classical Banach Spaces I, Springer-Verlag, Berlin, 1977, Proposition 1.c.3] asserts the existence of norm-one vectors $x_{1}, \ldots, x_{n} \in E$ and norm-one functionals $f_{1}, \ldots, f_{n} \in E^{*}$ with $f_{i}\left(x_{j}\right)=\delta_{i j}$. Easy calculations show that

$$
\max _{i=1}^{n}\left|\alpha_{i}\right| \leq\left\|\sum_{i=1}^{n} \alpha_{i} x_{i}\right\| \leq \sum_{i=1}^{n}\left|\alpha_{i}\right| \text { for all } \alpha_{1}, \ldots, \alpha_{n} \in \mathbb{R}
$$

Identifying $E$ with $\mathbb{R}^{n}$, we then have $\|\cdot\|_{\infty} \leq\|\cdot\| \leq\|\cdot\|_{1}$. Thus if $X$ is a compact, connected subset of some $n$-dimensional normed space, with diameter one, then $X$ is isometric to a metric space of the form $(Y, v)$, where $y \in H\left(I^{n}\right)$ and $\nu_{\infty} \leq \nu \leq \nu_{1}$.

Now $N=\left\{v: v_{\infty} \leq v \leq v_{1}\right\}$ is a compact subset of $C\left(I^{n}\right) ;$ by the 
Ascoli-Arzela theorem. It is routine to show that the maps

$a: H\left(I^{n}\right) \times N \rightarrow \mathbb{R}$ and diam $: H\left(I^{n}\right) \times N \rightarrow \mathbf{R}$ are continuous. It follows from compactness that

$$
k_{n}=\sup \left\{a(X, v):(X, v) \in H\left(I^{n}\right) \times N \text { and } \operatorname{diam}(X, v)=1\right\}
$$

is strictly less than one.

We also note that $k_{n} \geq 1-2^{-n}$. To see this, give $\mathbf{R}^{n}$ the norm $\|\cdot\|_{\infty}$, and consider the subset

$X=\left\{\left(\alpha_{1}, \ldots, \alpha_{n}\right\}: 0 \leq \alpha_{i} \leq 1\right.$ for all $i$,

and $0<\alpha_{i}<1$ for at most one value of $i$.

\section{References}

[1] 0. Gross, "The rendezvous value of a metric space", Advances in game theory, 49-53 (Annals of Mathematics Studies, 52. Princeton University Press, Princeton, New Jersey, 1964).

[2] Felix Hausdorff, Set theory, and edition (Chelsea, New York, 1962).

[3] Ed. Helly, "Über Mengen knovexer Körper mit gemeinschaftlichen Punkten", Deutsche Math.-Ver. 32 (1923), 175-176.

[4] Richard B. Holmes, Geometric functional analysis and its applications (Graduate Texts in Mathematics, 24. Springer-Verlag, New York, Heidelberg, Berlin, 1975).

[5] Sidney A. Morris and Peter Nickolas, "On the average distance property of compact connected metric spaces", Arch. Math. (to appear).

[6] Hukukane Nikaidô, "On von Neumann's minimax theorem", Pacific $J$. Math. 4 (1954), 65-72.

[7] Wolfgang Stadje, "A property of compact connected spaces", Arch. Math. (Basel) 36 (1981), 275-280.

[8] John Strantzen, "An average distance result in Euclidean n-space", Bull. Austral. Math. Soc. 26 (1982), 321-330. 
[9] E. Szekeres and G. Szekeres, "The average distance theorem for compact convex regions", Bull. Austral. Math. Soc. (to appear).

[10] D.J. Wilson, "A game with squared distance as payoff", Bull. Austral. Math. Soc. (to appear).

Department of Pure Mathematics,

La Trobe University,

Bundoora,

Victoria 3083,

Australia. 